\title{
Resonant Guided Wave Networks
}

\author{
Eyal Feigenbaum, Stanley P. Burgos and Harry A. Atwater \\ Thomas J. Watson Laboratory of Applied Physics \\ California Institute of Technology Pasadena, CA
}

USA

\section{Introduction}

In the last two decades, the development of new photonic material design paradigms has opened up new avenues for designing photonic properties based on different underlying physics. For example, photonic crystals, as described elaborately throughout this book, are based on dispersive Bloch wave modes that arise in periodic index structures. Different in operation than photonic crystals, metamaterials (Smith 2004, Shalaev 2007) are based on subwavelength resonant elements (or "meta-atoms") that interact with incident radiation to give rise to complex refractive indices. In this chapter, we introduce a new approach to optical dispersion control based on resonant guided wave networks (RGWNs) in which power-splitting elements are arranged in two- and three-dimensional waveguide networks.

A possible framework for comparing and classifying photonic design paradigms is according to their basic resonating elements with which light interacts to give the desired artificial dispersion. Under this classification scheme, we can think of materials that operate based on the local interaction of waves with sub-wavelength resonating elements (i.e. metamaterials), structures based on the nonlocal interference of Bragg periodic waves (i.e. photonic crystals), and arrays of coupled resonator optical waveguides (CROWs) where adjacent resonators are evanescently coupled (Yariv 1999). Different from these existing concepts, the dispersion that arises in RGWNs is a result of the multiple closed-path loops that localized guided waves form as they propagate through a network of waveguides connected by wave splitting elements. The resulting multiple resonances within the network give rise to wave dispersion that is tunable according to the network layout. These distinctive properties, that will be described here, allow us to formulate a new method for designing photonic components and artificial photonic materials.

A RGWN is comprised of power splitting elements connected by isolated waveguides. The function of the splitting element is to distribute a wave entering any of its terminals between all of its terminals, as illustrated in Fig. 1a. The waves are then propagated in isolated waveguides between the splitting elements, where the local waves from different waveguides are coupled together. For example, four splitting elements arranged in a rectangular network layout form a $2 \times 2$ RGWN (see Fig. 1b). When one of the terminals is excited, the multiple splitting occurrences of the incident wave within the network form closed path resonances that reshape the dispersion of the emerging waves according to the network layout and is different from the dispersion of the individual waveguides. Properly 
designing this network layout reshapes the interference pattern and the optical function of the RGWN, as will be exemplified later in this chapter. The $2 \times 2$ RGWN consists of one closed loop resonance, however larger two- or three-dimensional networks can support multiple resonances, which give rise to more design possibilities.
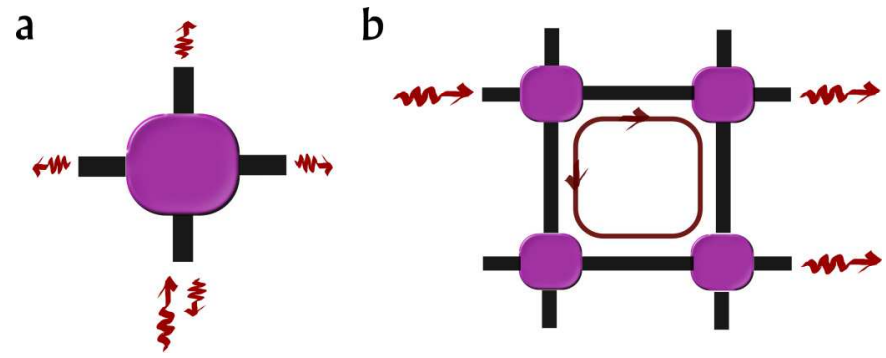

Fig. 1. Schematic illustration of (a) a 4-terminal equal power-splitting element and (b) a local resonance in a $2 \times 2$ RGWN.

Although the concept of RGWNs is quite general, we will first illustrate the underlying physics of this paradigm using plasmonics since it allows for a simple topological implementation. After introducing this implementation, in the following sections we will demonstrate how the local wave interference can be designed to engineer small (2x2) energy storage RGWN resonators, and also how we can program the optical transmission function of inhomogeneous RGWNs using transfer matrix formalism. We will also address how the same design principles can be utilized to control the optical dispersion properties of infinitely large RGWNs that behave like artificial optical materials. After addressing other possible implementation and practical issues we will conclude with possible future directions and a more detailed comparison to other optical design paradigms.

\section{Plasmonic RGWN components}

The operation of RGWNs is based on two basic components: power splitting elements and isolated waveguides. While the waveguides could easily be implemented using dielectric waveguides, the power splitting elements at the intersection of two such waveguides could not be achieved using dielectrics alone. Nevertheless, this splitting operation, which is the key enabler of this technology, is native to the intersection of two plasmonic waveguides. Consequently, a possible implementation of a RGWN is by using plasmonics via a mesh of intersecting sub-wavelength air gaps in a metal matrix.

Surface plasmon polaritons (referred here to as plasmons for brevity) are slow surface waves that propagate at metal-dielectric interfaces. Adding another metal-dielectric interface to this system, results in a metal-insulator-metal (MIM) waveguide, which supports a highly confined plasmon wave (the lowest order transverse magnetic mode TM0) that does not get structural cut-off as the dielectric gap between the metal layers becomes vanishingly small. The existence of this lowest-order plasmonic mode in MIM waveguides allows for such plasmonic components as power splitters (Feigenbaum 2007-1) and high transmission sharp waveguide bends (for a review of MIM waveguides and their possible applications see Feigenbaum 2007-2). However, the existence of metal in the MIM 
waveguide configuration does add a source of a modal attenuation to the system as a result the usual loss mechanisms present in any real metal-containing system. This results in a trade off between the compression of the modal cross-section and the modal attenuation as the air gap size is decreased. Since the loss in metals is strongly frequency and material dependent, the focus here will be on RGWNs composed of Au-air-Au MIM waveguides operating at telecommunication frequencies where the modal propagation lengths are on the order of tens of microns, which are substantially larger than the propagation lengths at visible frequencies. The optical properties of the materials throughout this chapter are based on tabulated data (Palik 1998).

In this implementation, the intersection of two sub-wavelength MIM waveguides forms an $X$-junction that functions as the power splitting elements in the network (Feigenbaum 20071) and the MIM segments between the intersections serve as the isolated waveguides connecting the $\mathrm{X}$-junctions. Through this implementation, $\mathrm{X}$-junctions can be tuned to split power equally at infrared wavelengths both for continuous waves and for short pulse waves consisting of only a few optical cycles while conserving the shape of the input signal. The observed equal-power split is a result of the subwavelength modal cross-section of the input plasmonic waveguide that excites the junction with a broad spectrum of plane waves. As such, equal four-way optical power splitting is enabled for transmission lines (e.g., MIM and coaxial configurations) but cannot be easily achieved using purely dielectric waveguides due to their half-wavelength modal cross-section limit. Thus, through a plasmonic implementation, the strong coupling to all four neighboring $X$-junctions gives the plasmonic RGWN structure an optical response different from a cross-coupled network of purely dielectric waveguides, where most of the power would be transmitted in the forward direction, with only weak coupling to perpendicular waveguides.
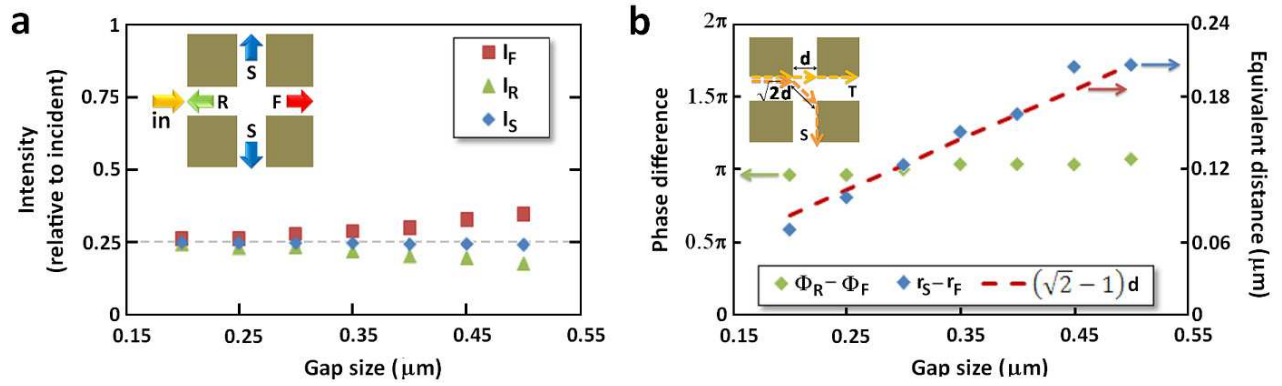

Fig. 2. Power splitting properties of the emerging pulses in an $X$-junction: (a) intensity relative to the exciting pulse, and (b) phase difference at $\lambda_{0}=1.5 \mu \mathrm{m}$ (Feigenbaum 2010).

As the MIM waveguide air gap thickness is varied, the power-split between the X-junction terminals can be tuned both in terms of amplitude and phase (Feigenbaum 2010). This, in addition to determining the phase accumulation in the waveguide segments, sets independent controls in designing the interference pattern that governs the operation of a RGWN. The power splitting in the Au-air X-junction was investigated using the 2D finitedifference time-domain (FDTD) method with short pulse excitation and two equal thickness intersecting MIM waveguides. Through this study, it was found that for small $(0.25 \mu \mathrm{m})$ MIM gaps, these plasmonic X-junctions exhibit equal power splitting with the reflected 
pulse being out-of-phase (i.e., approximately ח-phase shifted) with respect to the sideways and forward transmitted pulses. As illustrated in Fig. 2a, as the MIM gap size is increased, the optical power flow deviates from equal power splitting between the terminals towards dominant power transmission directly across the X-junction, which resembles the wavelength-scale photonic mode limit. Furthermore, in these calculations, the phase shift between the sideways (S) and the forward (F) transmitted pulses is consistent with the geometrical difference in their pulse propagation trajectories (see Fig. 2b).

\section{Resonators}

After characterizing the properties of the RGWN building blocks, we illustrate the working principles of RGWNs by investigating the dynamics of a compact $2 \times 2$ RGWN resonator. In order to form a resonance, the network is designed such that when an X-junction is excited from the internal ports, the exciting waves are out-of-phase, resulting in constructive interference inside the network, as illustrated in Fig. 3. For such out-of-phase excitation the fields in the external terminals interfere destructively, and the power is coupled back into the resonator, enhancing the energy storage quality factor (Q-factor).
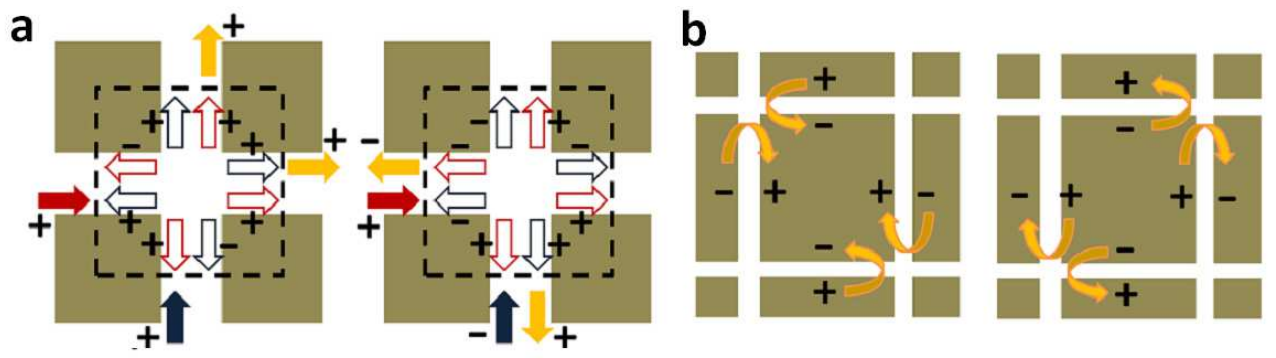

Fig. 3. Resonance build-up in a $2 \times 2$ RGWN. (a) Two in/out-of phase input pulses result in destructive/constructive interference inside the network. (b) Steady-state of waves resonating in a $2 \times 2$ network where each pair of pulses excites the $X$-junctions out of phase (Feigenbaum 2010).

When the $2 \times 2$ RGWN is excited from the lower-left arm (see Fig. 4), after a transient that includes the first five splitting events, the resonant state is reached as pairs of pulses resonate between junctions 1 and 3 (exemplified by snapshot $t_{6}$ ) and junctions 2 and 4 (exemplified by snapshot $t_{7}$ ). However, before this steady state is reached, it is instructive to follow the dynamics that lead up to this resonance. Starting with the third power split, this event occurs as junctions 1 and 3 are both simultaneously excited by two waveguides. The incoming pulses arrive at both junctions in-phase, which would result in destructive interference inside the network if the $\mathrm{R}$ and $\mathrm{S}$ split components of each pulse were exactly $\Pi$-phase shifted. However, the interference is not completely destructive due to the finite size of the waveguides, which causes the phase difference to deviate from a perfect ח-phase shift (in accordance with Fig. 2). This power splitting event determines how much power couples into the network. For all future power splitting events after the third one, the two pulses arriving simultaneously at each junction are out-of-phase and therefore interfere constructively inside the resonator. The trade-off between coupling power into the resonator 
and maintaining it inside suggests that MIM gap sizes that are subwavelength, but not arbitrarily small, will maximize the network resonance. To interpret the FDTD observations and arrive to the conclusion described above, a simplified analytical description of pulse propagation in the network is derived in which only a few parameters are tracked: phase, amplitude, position and direction. The pulses are assumed to travel in the waveguides and split into four new pulses upon arrival at an X-junction. This model also illustrates the compactness of the possible mathematical representation of RGWNs, and the importance of this advantage becomes more substantial when considering the dynamics of larger $2 \mathrm{D}$ and 3D network topologies.
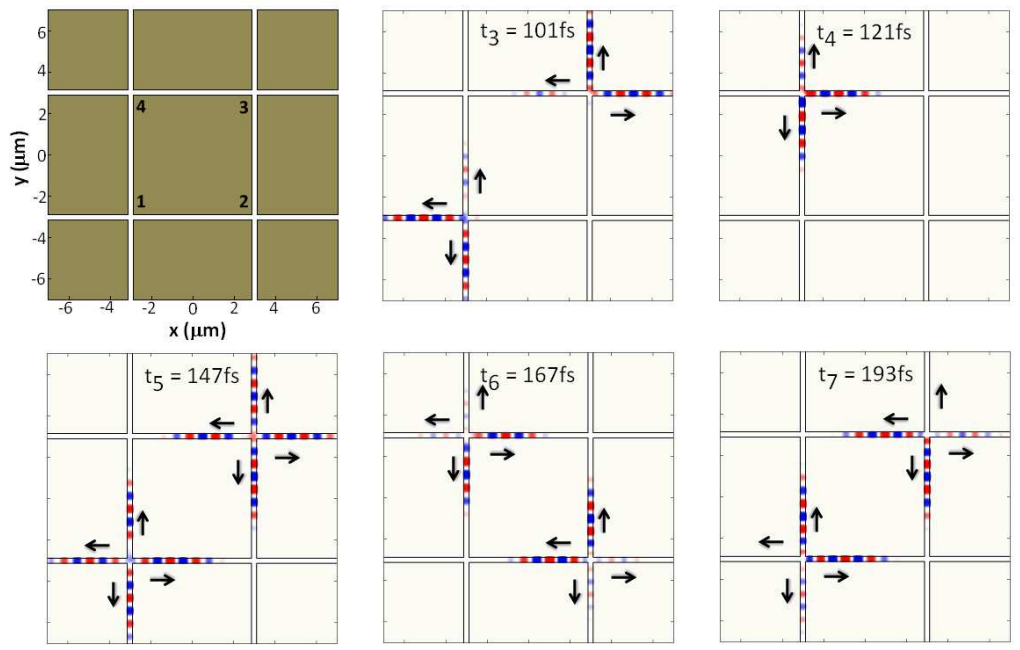

Fig. 4. Time snapshots of $H_{z}$ (normalized to the instantaneous maximum value) in a $2 \times 2$ plasmonic RGWN recorded at the third to the seventh power splitting events for a 2D-FDTD simulation. The MIM waveguides are $0.25 \mu \mathrm{m}$ thick and $6 \mu \mathrm{m}$ long (Feigenbaum 2010).

Calculating the Q-factor of such $2 \times 2$ RGWN resonators (Fig. 5) illustrates the role of interference in generating a strong network resonance, which causes the network Q-factor to be an order of magnitude larger than what would be expected if optical power splitting in the X-junctions operated incoherently, i.e. we lost half the power in each splitting event. Increasing the MIM gap size causes the phase of the interfering waves to deviate from being $\Pi$-phase shifted, resulting in a degradation of the constructive interference inside the resonator and a decrease in the overall network Q-factor. On the other hand, as the gap size is decreased, the plasmonic mode attenuation increases due to metallic losses in the waveguides. Between these two competing effects, the maximal Q-factor value is obtained for a gap size of $250 \mathrm{~nm}$. These RGWN Q-factor values are considerable for plasmonic resonators and even comparable to typical values of wavelength-size dielectric resonators that are dominated by radiation loss (e.g., a cylindrical dielectric cavity of radius $1.3 \lambda$ with a purely real refractive index of $n=2.5$ surrounded with air has a $Q \sim 100$ ). If we were to artificially decrease the $\mathrm{Au}$ loss at $1.5 \mu \mathrm{m}$ (or alternatively go to longer wavelengths), the Qfactor of the resonator would increase appreciably (e.g., $Q \sim 750$ for a $200 \mathrm{~nm}$ gap width), indicating that the resonator $\mathrm{Q}$-factor is primarily limited by the material loss. 


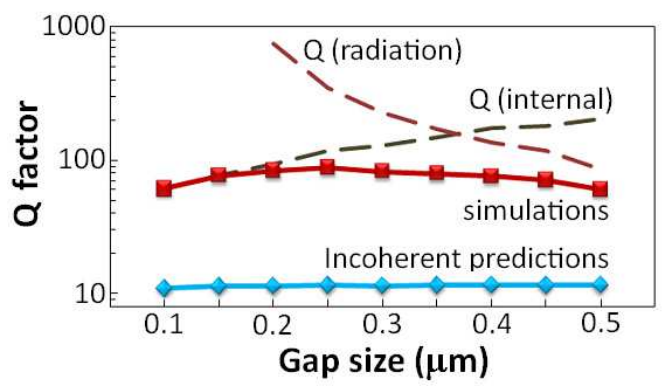

Fig. 5. Q-factor of $2 \times 2$ RGWN resonator from simulation results compared with those resulting from incoherent power splitting (Feigenbaum 2010).

\section{Tailoring the optical properties of artificial materials}

After studying the resonance effects in a small RGWN, we now investigate the dispersion characteristics of infinitely large 2D periodic RGWNs by modelling the structure unit cell in FDTD with Bloch boundary conditions. Through this analysis, we find that RGWNs exhibit wave dispersion and photonic bandgaps due to interference effects, and that their band structure can be controlled by modifying the network structural parameters. Two different length-scales control the network dispersion: the subwavelength width of the MIM gaps determines the phase shift at each X-junction, and the wavelength-order distance between the nodes along with network topology determine the interference scheme.
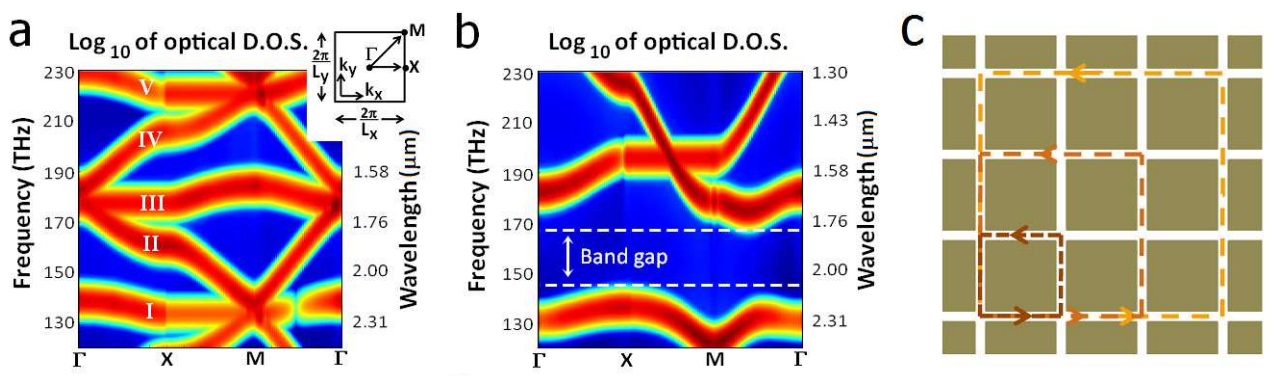

Fig. 6. Photonic band structure of infinitely large periodic RGWNs (Feigenbaum 2010).

The same interference dynamics that govern the energy storage in finite-size $2 \times 2$ RGWN resonators are the same that control the optical properties of artificially designed RGWN materials of infinite-size. If the network parameters are chosen such that a planewave excitation at a given incidence angle results in a resonance effect similar to the one demonstrated for the $2 \times 2$ network, then this would correspond to a forbidden state of propagation in the photonic band diagram. Examining the optical density of states (DOS) for different wave vectors over frequencies in the near infrared range, where the material (Au in this case) dispersion is small, we observe a photonic band structure which is only due to dispersion resulting from the network topology, as shown in Fig. 6a. The functionality of the infinitely large RGWN is not hindered by loss since its dispersion depends on the waveguide decay length being much larger than the size of the largest resonant feedback 
loop that has dominant contribution to the RGWN dispersion. Further possibilities for achieving band dispersion control are illustrated in Fig. 6b, showing a flat bands over a wide range of wavevectors at 130 and $170 \mathrm{THz}$, as well as the formation of a photonic bandgap between 140-160 THz, for appropriately chosen network parameters.

The infinitely large RGWN is illustrated in Fig. $6 c$ along with a few schematic resonance orders that represent the resonances that could arise within the network. The operating mechanism of the RGWN is very different from that of photonic crystals composed of metaldielectric alternating materials. Although the schematic layout might look similar, the difference between the two classes of artificially designed optical materials becomes clear when considering the difference in the length scales of their composite elements. Whereas photonic crystals operate based on non-local interaction of Bloch waves with the entire array, RGWNs rely on the interference of local waves. Therefore RGWNs are not sensitive to the actual topology of waveguides between junctions but only to its trajectory length, whereas the properties of photonic crystals would greatly depend on the shape of the periodic metallic islands. Additionally, RGWNs do not necessarily have to be periodic to operate as resonant guided wave networks, and for the same reason, planting a defect in a RGWN would not have the same effect as it would in a photonic crystal.

\section{Programming the optical properties of a network}

Because the underlying physics of RGWNs is based on the interference of local waves, it allows for layouts that are inhomogeneous and non-periodic across the network. Unlike photonic crystals, which are restricted to Bragg wave effects in periodic structures, the flexibility of RGWNs open up design possibilities where the wave properties are varied across the structure. With respect to metamaterials, which could inherently be nonhomogeneous due to the local nature of the interaction between light and the meta-atoms, RGWNs have the advantage of having interference effects within the network, which allows for frequency spectrum reshaping designs through these effects.

An additional unique feature of RGWNs relates to the constraints on wave propagation within the structure. Unlike other photonic designs, RGWNs have a limited number of modes that are allowed to propagate within the structure (e.g. only the TM0 mode for the case of the plasmonic implementation described previously). Furthermore, the waves can propagate only inside the waveguides connecting the splitting elements. The different waveguides are coupled only by X-junctions, which each have only a limited number of terminals. This level of control is beneficial for several reasons. First, the interference pattern in the network can be controlled more directly. Second, it allows for a comprehensive mathematical representation of the RGWN by scattering matrix (S-matrix) formalism that greatly reduces the computational complexity of programming the network. Third, since the waveguides are isolated from each other, their only contribution to the network is to serve as phase retardation elements between the splitting elements. As a result, the waveguide length is the only effective parameter in its contour, as long as the bending is not too severe. This waveguide feature allows for the network to maintain its engineered function even when distorted. Additionally, the ability to utilize curved or bent waveguides to accommodate long contours is useful when designing the interference pattern of RGWNs. 
These distinctive RGWN characteristics open up new opportunities for designing photonic devices by programming the entire network rather than by assembling interconnected discrete components with traceable functions. The usual way of designing photonic devices is to target the desired subsystem functions, map them logically into sub-functions, and then assembling components that carry out these sub-functions in the desired system. For example, a wavelength router could be designed using add/drop ports where the input and output waveguides are coupled by wavelength sensitive ring resonators (Little 1997) or by defects in a photonic crystal (Fan 1998). Similarly, in free space optics, this function could be achieved through the use of collinear beam splitters, each designed to deflect a desired wavelength band. In these schemes, the couplers and waveguides are discrete components that are associated with a specific function, and are combined in a logical way to carry out the overall system function. An alternative approach is to use a network of components that carries out the desired function but, unlike traditional designs, there is no specific logical sub-function associated with any individual component. While the inner connectivity of the device will be less intuitive, it has the potential to result in more efficient designs of complex and compact devices.

One possible way of representing a system function in a RGWN is through the use of a scattering operator that maps the set of local waves entering the device terminals to the set of the waves exiting from the same terminals (Feigenbaum 2010-2). Since a RGWN is composed of a discrete set of components (waveguides and X-junctions) and terminals, the system function is represented by a scattering matrix (S-matrix) connecting the vectors of the waves entering and emerging from the RGWN via the external ports (see Fig. 7). Designing the system function of the RGWN is then mathematically equivalent to designing the S-matrix to yield a desired output, given a set of inputs.
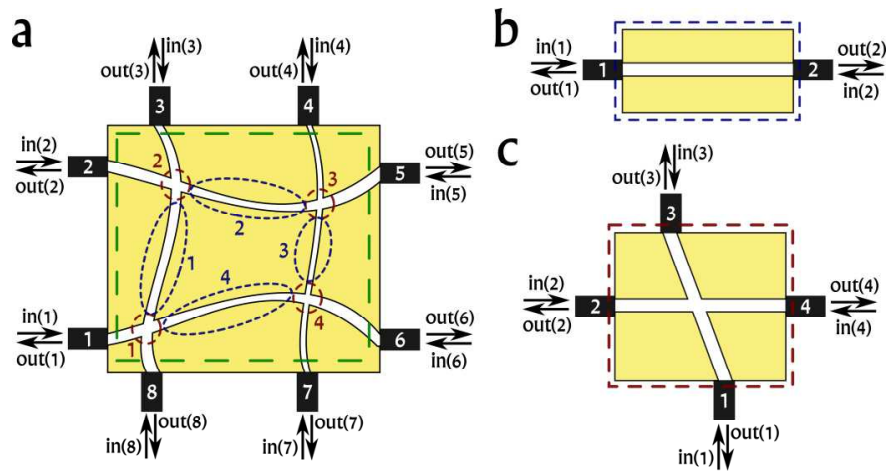

Fig. 7. Mathematical representation scheme of (a) a 2x2 RGWN system and its components, (b) a waveguide component, and (c) an X-junction component (Feigenbaum 2010-2).

Programming an optical function onto a network, according to the design principle described above, will first be demonstrated for a plasmonic $2 \times 2 \mathrm{RGWN}$, in which the constituent MIM waveguides are allowed to differ in width, length, and contour. The device has eight terminals, numbered from ' 1 ' to ' 8 ', as illustrated in Fig. $7 \mathrm{a}$. The input vector lists the complex amplitudes of the magnetic fields (H-fields) entering the network in the eight terminals, and similarly the output vector describes the complex $\mathrm{H}$-field amplitudes of the waves exiting the network through these same terminals. 
The network S-matrix is assembled from the mathematical representation of its components according to the network layout. As a first step, a 'function library' of mathematical representations is generated for all the possible network components (i.e., waveguides and X-junctions) using finite difference time domain (FDTD) full wave electromagnetic simulations. Once this library is established, the RGWN S-matrix can be assembled according to the network layout. It is worth pointing out that the S-matrix calculation scheme is almost always found to be much faster than resolving the RGWN behavior from full wave electromagnetic simulations, yet reproduces the same information about the network. This becomes significant for optimization tasks and especially as the network size increases.

To carry out this formalism, the two basic RGWN components (waveguides and Xjunctions) need to first be represented mathematically. The waveguides are mathematically represented by their complex phase retardation, determined by the complex propagation constant of the wave and the waveguide length. The propagation constants are extracted from FDTD simulations for waveguides with various widths at different frequencies. The $\mathrm{X}$-junctions, which are comprised of two intersecting waveguides with four terminals, are mathematically represented by a $(4 \times 4)$ S-matrix. For a given set of waveguide widths, the complex transmission coefficients of the $\mathrm{X}$-junction ports are extracted from FDTD simulations by measuring the amplitude and phase of the wave transmitted to the different ports when excited from one of the terminals at a given wavelength.

The S-matrix of the $2 \times 2$ RGWN is then assembled from the mathematical representation of its constituent components according to the network layout. The phasor representation of the local wave $\mathrm{H}$-fields in the network is represented by three column vectors (transposed for brevity):

$$
\begin{aligned}
& \underline{A}_{\text {out }}{ }^{t r}=\left\{a_{1(1)}^{o}, a_{1(2)}^{o}, a_{2(3)}^{o}, a_{2(2)}^{o}, a_{4(1)}^{o}, a_{4(4)}^{o}, a_{3(3)}^{o}, a_{3(4)}^{o}\right\} ; \\
& \underline{A}_{\text {in }}{ }^{t r}=\left\{a_{1(1)}^{i}, a_{1(2)}^{i}, a_{2(3)}^{i}, a_{2(2)}^{i}, a_{4(1)}^{i}, a_{4(4)}^{i}, a_{3(3)}^{i}, a_{3(4)}^{i}\right\} ; \\
& \underline{A}_{\text {net }}{ }^{t r}=\left\{a_{1(3)}^{i}, a_{1(4)}^{i}, a_{2(1)}^{i}, a_{2(4)}^{i}, a_{4(3)}^{i}, a_{4(2)}^{i}, a_{3(1)}^{i}, a_{3(2)}^{i}\right\} ;
\end{aligned}
$$

where $A_{\text {out }}$ and $A_{\text {in }}$ hold the values of the local input and output waves of the RGWN at its ports, and $A_{\text {net }}$ represents the input wave on the X-junctions from the internal terminals of the RGWN. The i/o superscripts denote input/output waves with respect to the X-junction, the number subscripts corresponds to the junction number as defined in Fig. 7a, and the bracketed number subscripts label the ports as defined in Fig. 7c. The coupling of the H-field vectors by the network connectivity can then be represented by the system:

$$
\left\{\begin{array}{c}
\underline{A}_{\text {out }}=\underline{\underline{M}}_{F S} \underline{A}_{n e t}+\underline{\underline{M}}_{R S} \underline{A}_{i n} \\
\underline{0}=\left(\underline{\underline{M}}_{R S}-\underline{K}\right) \underline{A}_{n e t}+\underline{\underline{M}}_{F S} \underline{A}_{i n}
\end{array}\right.
$$

where $M_{F S}$ and $M_{R S}$ are diagonal 8-by-8 matrices that originate from the splitting relations in the $\mathrm{X}$-junctions and $K$ is a sparse 8 -by- 8 matrix that stands for the wave propagation in the waveguides. These matrices are defined as: 


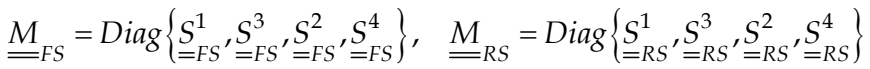

$$
\begin{aligned}
& \underline{S}_{F S}^{i}=\left(\begin{array}{cc}
\left(t_{F}^{V}\right)^{i} & \left(t_{S}^{H}\right)^{i} \\
\left(t_{S}^{V}\right)^{i} & \left(t_{F}^{H}\right)^{i}
\end{array}\right) \quad \underline{S}_{R S}^{i}=\left(\begin{array}{cc}
\left(t_{R}^{V}\right)^{i} & \left(t_{S}^{H}\right)^{i} \\
\left(t_{S}^{V}\right)^{i} & \left(t_{R}^{H}\right)^{i}
\end{array}\right) \\
& \underline{K}=\left\{\begin{array}{c}
K(1,3)=K(3,1)=\kappa_{1}^{3} \\
K(2,6)=K(6,2)=\kappa_{1}^{2} \\
K(4,8)=K(8,4)=\kappa_{3}^{4} \quad, \kappa_{i}^{m}=\exp \left\{j(\beta L)_{i \Leftrightarrow m}\right\} \\
K(5,7)=K(7,5)=\kappa_{2}^{4} \\
\text { other matrix elements }=0
\end{array}\right.
\end{aligned}
$$

where the $\mathrm{V} / \mathrm{H}$ superscript index denotes if the transmission coefficient is for excitation of the vertical or the horizontal waveguide of that $\mathrm{X}$-junction.

Algebra of equation set 3 gives the matrix representation of the 2x2 RGWN S-matrix:

$$
\underline{\underline{S}}^{2 \times 2 R G W N}=\underline{\underline{M}}_{R S}-\underline{\underline{M}}_{F S}\left(\underline{\underline{M}}_{R S}-K\right)^{-1} \underline{\underline{M}}_{F S}
$$

When validating the field amplitude predictions of the S-matrix representation with FDTD simulations, less than $5 \%$ difference is found for various test cases. The two major contributions to this small deviation result from the interpolation between the parameter space points, where the library components were calculated, and from the error added when the waveguides are bent. For cases where no interpolation or waveguide bending occurs, the FDTD results differ by only $1 \%$ from the S-matrix predictions. The ability to accurately predict the RGWN interference using S-matrix representation reduces the complicated task of programing a desired optical function into a RGWN into an efficient optimization of its Smatrix.

For example, the RGWN can be programmed by minimizing the difference between the actual network output and the desired one (for a given input), as the network parameter space is swept across the various waveguide widths and lengths. The optimization process then results in a set of network parameters that can be translated to a network layout and then validated with FDTD simulations.

\section{Multi-chroic filters using RGWNs}

The S-matrix programming method can be exemplified by designing a $2 \times 2 \mathrm{RGWN}$ to function as a dichroic router (Fig. 8a). Although simple in concept, the exercise of setting a passive device to have different functions at different wavelengths is quite instructive. Explicitly, the required function is to route two different wavelengths $\left(\lambda_{1}\right.$ and $\left.\lambda_{2}\right)$ to a different set of ports (' 1 ' and ' 6 ' for $\lambda_{1}$ and ' 2 ' and ' 5 ' for $\lambda_{2}$ ) when the two bottom ports (' 7 ' and ' 8 ') are simultaneously excited with equal power. Mathematically, we can represent the device as an $8 \times 8$ S-matrix $\mathbf{S}\left(\lambda_{1}, \lambda_{2}\right)$ connecting the input and the output vectors. For both wavelengths, the input vector is nonzero for the bottom ports (i.e. $\mathbf{I n}=(0,0,0,0,0,0,1,1))$ and the desired output vectors would be $\operatorname{Out}\left(\lambda_{1}\right)=(1,0,0,0,0,1,0,0)$ for $\lambda_{1}$ and $\operatorname{Out}\left(\lambda_{2}\right)=(0,1,0,0,1,0,0,0)$ for $\lambda_{2}$. Because we 
do not have enough degrees of freedom in this small $2 \times 2$ network to exactly attain the desired outputs, we instead optimize the ratio of power going to the two sets of ports at the different wavelengths.

a

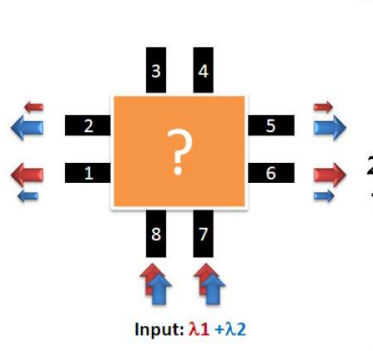

b

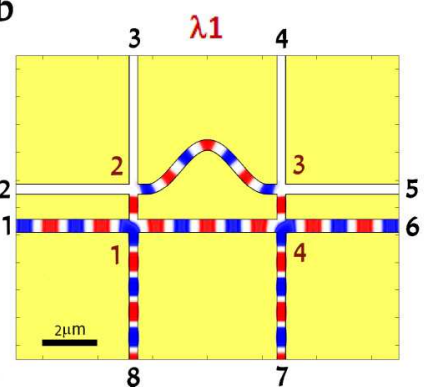

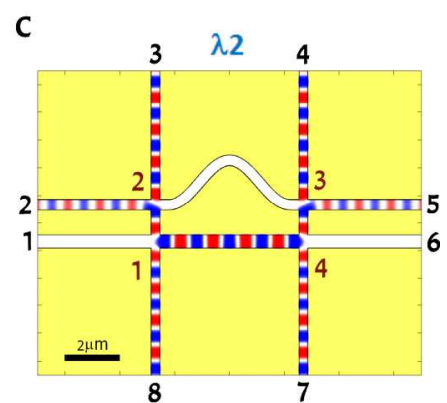

Fig. 8. 2x2 RGWN programmed to function as a dichroic router: (a) schematic drawing, and (b, c) time snapshots of the H-field at the two operation frequencies (Feigenbaum 2010-2).

The optimization procedure is implemented in Matlab using the pre-calculated mathematical representation data set of the RGWN components obtained from full-field electromagnetic FDTD simulations excited with continuous wave sources (see illustration in Fig. 9).

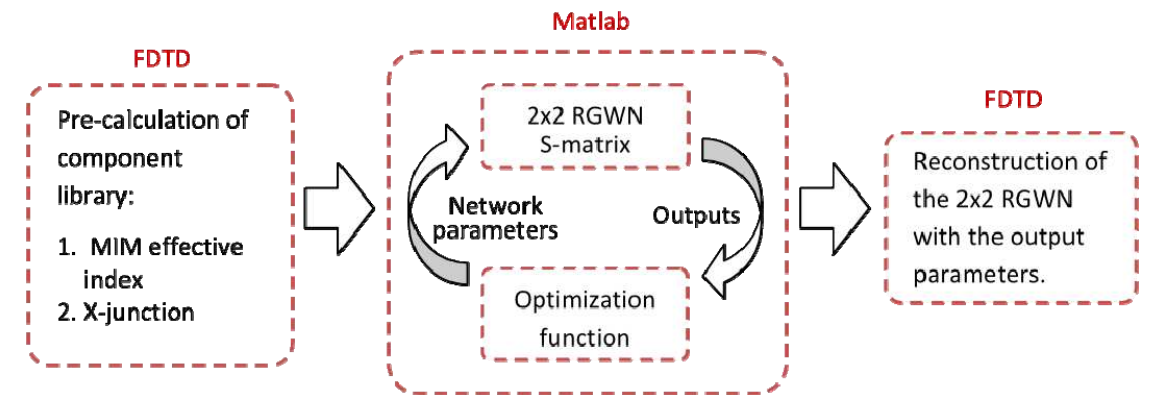

Fig. 9. Flow chart of the RGWN S-matrix optimization procedure (Feigenbaum 2010-2).

The dichroic router network is defined by eight parameters: the length and width of the upper, lower, and side waveguides and the two wavelengths. The waveguide widths determine the effective index in the waveguides as well as the transmission coefficients of the X-junctions. The optimization procedure is conducted in Matlab with the optimization function for the dichroic router defined as follows:

$$
\begin{aligned}
& O_{1}=\left|\operatorname{Out}_{6}\left(\lambda_{1}\right)\right| /\left|\operatorname{Out}_{5}\left(\lambda_{1}\right)\right| \\
& O_{2}=\left|\operatorname{Out}_{5}\left(\lambda_{2}\right)\right| /\left|\operatorname{Out}_{6}\left(\lambda_{2}\right)\right| \\
& \frac{1}{f}=\left[\sqrt{\left|O u t_{6}\left(\lambda_{1}\right)\right| \cdot\left|O u t_{5}\left(\lambda_{2}\right)\right|}\right] \cdot\left[O_{1} \cdot O_{2}\right] \cdot\left[1-\left|\frac{O_{1}-O_{2}}{O_{1}+O_{2} \mid}\right|\right]
\end{aligned}
$$

where $O_{1}$ and $O_{2}$ represent the two terminal output ratios that need to be maximized at the two different wavelengths, $\lambda_{1}$ and $\lambda_{2}$. The function $f$ is used to merge the two ratios together 
into one weighted optimization parameter, where the first term in squared brackets maximizes the total power routed into the selected terminals, the second term maximizes the two ratios, and the third term is a weighting factor that ensures that the two ratios are maximized equally. The target function is defined as the inverse of these three terms in multiplication for the minimization Matlab function. At each point in the parameter space, the network output vector is calculated as the multiplication of the $2 \times 2$ RGWN S-matrix evaluated at the parameter values times the input vector representing excitation only from the two bottom terminals (' 7 ' and ' 8 ').

After defining the optimization function, we constrain the parameter space based on practical considerations. The parameter space includes the width and length of the upper, lower, and side waveguides as well as the two wavelengths of operation $\left(\lambda_{1}\right.$ and $\left.\lambda_{2}\right)$. We decrease the number of parameters to optimize by restricting the device to have left-right symmetry based on the desired operation. We restrain the design to operate in the infrared frequency range $\left(\lambda_{0}=1.2-2 \mu \mathrm{m}\right)$ where the material dispersion and loss are less pronounced than in the visible. Furthermore, the waveguide thickness is constrained to be small enough to only support the lowest order plasmonic mode (air gap widths 100-500nm).

The optimization procedure yields the network parameters given in Table 1, which reveal that the required RGWN for color routing is distributed inhomogeneously.

\begin{tabular}{|l|l|l|}
\hline Waveguides & Width $(\mu \mathrm{m})$ & Length $(\mu \mathrm{m})$ \\
\hline Lower & 0.47 & 5.4 \\
\hline Side & 0.31 & 1.34 \\
\hline Upper & 0.38 & 6.6 \\
\hline
\end{tabular}

Table 1. Set of optimized parameters for $2 \times 2$ RGWN dichroic router operating at $\lambda_{1}=2 \mu \mathrm{m}$ and $\lambda_{2}=1.26 \mu \mathrm{m}$.

When translating the optimized network parameters into the network layout, we learn that the upper waveguide is longer than the lower one, and therefore needs to be bent. Importing the resulting layout into FDTD, we obtain the steady state $\mathrm{H}$-field distribution shown in Fig. 8b and 8c which show time snap shots at the two operation wavelengths. The FDTD simulation results validate the S-matrix design, with $\lambda_{1}$ and $\lambda_{2}$ clearly routed to a different set of sideways ports as illustrated in Fig. $8 b$ and $8 c$, respectively. From these FDTD results, it is also possible to observe the build-up of local resonance inside the network, which results in the filtering out of the desired output ports. We note that the transmission (' 3 ' and ' 4 ') and reflection $\left({ }^{\prime} 7{ }^{\prime}\right.$ and ' 8 ') ports from the device are not identically zero since the device does not have enough degrees of freedom and were therefore not included in the optimization function.

The matrix representation can also be used to understand the interference conditions through which the RGWN accomplishes its desired function. From the known input vector and the network S-matrix, the wave complex amplitudes can be identified at any point in the network. For each wavelength, we resolve the excitation conditions of the X-junctions that have the ports that are to be filtered out. For example, for $\lambda_{1}$ to be filtered out from terminals ' 2 ' and ' 5 ', we examine the excitation conditions in X-junction ' 3 ,' which has four terminals. Two of the terminals are external device ports (' 4 ' and ' 5 ') and the other two are internal network terminals. There is no input signal incident on the two external ports, so it 
is the excitation conditions of the remaining two junction terminals that null the output in terminal '5.' Indeed, the excitation amplitudes of junction ' 3 ' obtained from the S-matrix representation are $0.23 \exp (-\mathrm{j} 0.21 \Pi)$ and $0.34 \exp (\mathrm{j} 0.64 \Pi)$, which are close in amplitude and $\sim \Pi$ phase-shifted. This is consistent with the results from section 3, which show that when an 'X-junction' is simultaneously excited $\Pi$ phase-shifted from two adjacent terminals, the two other terminals will be filtered out (Fig. 3a). The fact that the excitations are not exactly the same in amplitude and $\Pi$ phase-shifted is attributed to the additional constraints the design has on the other wavelength as well as the limitations imposed on the parameter space.

Similarly, the excitation conditions necessary for filtering out terminals ' 1 ' and ' 6 ' at $\lambda_{2}$ (Fig. $8 b$ ) are examined by focusing on the S-matrix amplitudes of $X$-junction ' 4 .' In this case there are three terminals being excited: the lower terminal of the $\mathrm{X}$-junction (port ' 7 ') is given by the network excitation, so the excitation of the other two internal ports will determine the filtering out of port ' 6 .' Intuitively, the condition to filter out terminal ' 6 ' will be simply a $\Pi$ phase-shifted excitation of the upper and lower terminals of junction ' 4 ', with zero excitation from the side port. From the case of $\lambda_{1}$ we also know that additional constraints might cause a residual wave emerging from terminal ' 6 ', which could be compensated by a small amplitude excitation at the other side terminal of the junction ' 4 .' Indeed, the excitation amplitudes of junction ' 4 ' in the S-matrix representation are 1 in lower terminal, $0.9 \exp (\mathrm{j} 0.82 \pi)$ in upper terminal, and $0.3 \exp (-\mathrm{j} 0.32 \Pi)$ in the side terminal.

To further exemplify the programmability of RGWNs via S-matrix formalism, we consider a $3 \times 3$ RGWN programmed to function as a trichroic router. In order to implement the more complex task of routing three wavelengths we allow for more degrees of freedom in the network by increasing the number of components, effectively increasing the amount of data contained. The function is defined as an extension of the dichroic router, but here when the three bottom terminals are simultaneously excited at three different frequencies, the frequencies are filtered out to three different sets of side terminals as illustrated in Fig. 10. The analysis results in the optimal RGWN parameters shown in Table 2.

a

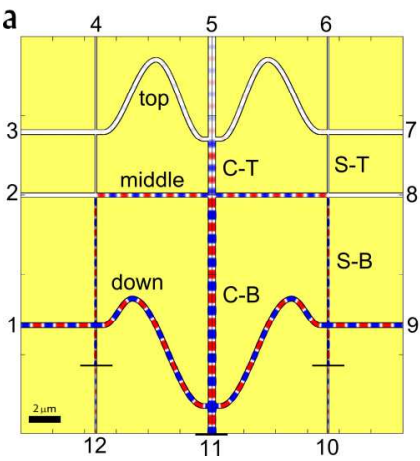

b

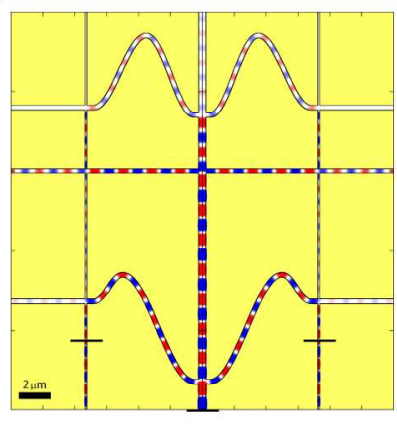

C

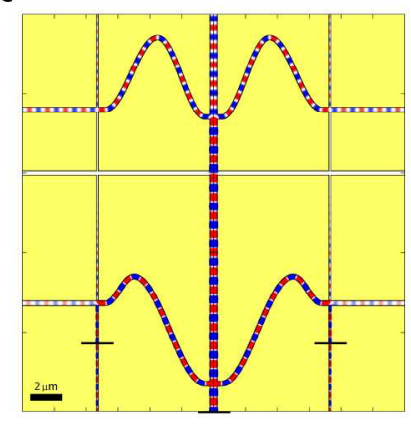

Fig. 10. 3×3 RGWN programmed to function as a trichroic router. Time snapshots of the steady state $\mathrm{H}$-field at the three operation frequencies (Feigenbaum 2010-2): a) $\lambda_{1}$, b) $\lambda_{2}$, c) $\lambda_{3}$.

It is interesting to note that the wavelengths are not mapped monotonically to the output terminals (i.e. from bottom/top ports as the wavelength increases/decreases), which would be the usual case for devices relying on material dispersion, such as a glass prism. 


\begin{tabular}{|c|c|c|c|}
\hline Waveguides & & $\begin{array}{c}\text { Width } \\
(\mu \mathrm{m})\end{array}$ & $\begin{array}{c}\text { Length } \\
(\mu \mathrm{m})\end{array}$ \\
\hline \multirow[t]{4}{*}{ Vertical } & center-bottom (C-B) & 0.45 & 13.25 \\
\hline & side-bottom (S-B) & 0.1 & 8.15 \\
\hline & center-top $(\mathrm{C}-\mathrm{T})$ & 0.45 & 3.55 \\
\hline & side-top (S-T) & 0.1 & 4 \\
\hline \multirow[t]{3}{*}{ Horizontal } & Top & 0.29 & 12.8 \\
\hline & Middle & 0.26 & 7.3 \\
\hline & Down & 0.3 & 11.95 \\
\hline
\end{tabular}

Table 2. Set of optimized parameters for a $3 \times 3$ RGWN trichroic router operating at $\lambda_{1}=1.59 \mu \mathrm{m}, \lambda_{2}=1.97 \mu \mathrm{m}$, and $\lambda_{3}=1.23 \mu \mathrm{m}$.

\section{Possible Implementations}

The underlying physics and the working principles of the RGWNs were demonstrated in the previous sections with an idealized 2D implementation using MIM waveguides. However, for the same 2D network topology as shown in Fig. 4, but implemented with 3D high aspect ratio Au-air channel plasmon waveguides (Bozhevolnyi 2006), the observed wave dynamics are found to closely resemble that of the 2D MIM waveguide network, as studied with 3D full-field simulations. If the aspect ratio of the channel plasmon waveguide is high enough, the propagating mode within the channels strongly resembles the MIM gap plasmonic mode. This can for instance be seen in the measured quality factors of RGWNs comprised of channel plasmon waveguides (3D simulations) and MIM slot waveguides (2D simulations) which have Q-factor values of 82 and 83, respectively, at a wavelength of $1.5 \mu \mathrm{m}$. Furthermore, the two power splitting events that define the RGWN resonant state are similar for both the channel and MIM waveguides (Fig. 4).
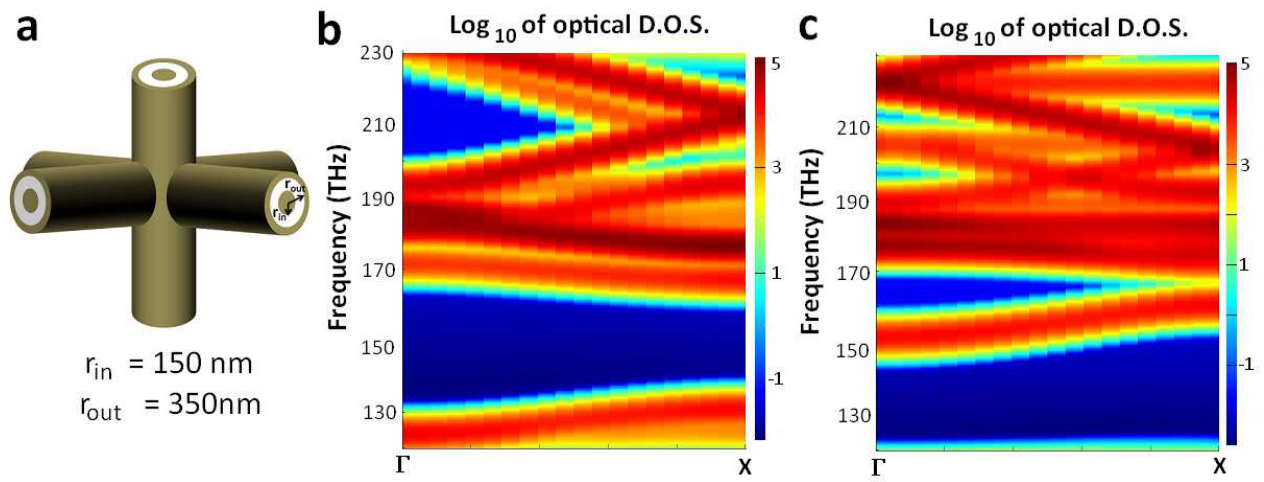

Fig. 11. 3D RGWN: (a) rendering of a 3D RGWN building block (6-arm junction). (b, c) Optical DOS of an infinite 3D network spaced periodically with cubic periodic unit cell with different spacing (Feigenbaum 2010-1).

The dispersion design in a volume can be addressed by 3D-RGWN topologies, for example, constructing an array of orthogonally intersecting 3D networks of coaxial Au-air waveguides aligned in a Cartesian grid (Fig. 11a). In this case, the four-arm X-junction 
element of the 2D network is replaced by a six-arm 3D junction element. Using 3D FDTD, we have verified that six-way equal power splitting occurs for pulsed excitation in a coaxial $\mathrm{Au}$-air waveguide junction. Like for the 2D-RGWN, the dispersion of the infinitely large periodic 3D-RGWN is predominantly determined by the network parameters rather than the waveguide dispersion. This is demonstrated by the noticeable difference in the band diagrams (Fig. 5b and 5c) obtained for two networks comprised of the same waveguides but with different inter-node spacing.

\section{Conclusions and future directions}

RGWNs offer a different approach for designing dispersive photonic materials. Whereas photonic crystals rely on the formation of Bloch wave states by interference of waves diffracted from an array of periodic elements, a truly non-local phenomenon; RGWNs rely on the coherent superposition of power flowing along isolated waveguides and splitting at $\mathrm{X}$-junctions. Furthermore, in photonic crystals, the interference pattern of the diffracted waves depends on the nonlocal periodic spatial arrangement of the diffracting elements; and in RGWNs it is the local network topology that determines the dispersion and resonance features. For example, in a RGWN, the coherent wave propagation through the network is determined only by the total path length along the waveguide and the phase shift added at a power splitting event, having no restriction on whether the waveguides are straight or curved. Metamaterials also feature a design approach based on the attributes of localized resonances, but their dispersive properties do not depend on any length scale between resonant elements - thus differing substantially from RGWNs. Arrays of coupled resonator optical waveguides (CROWs) feature discrete identifiable resonators that act as the energy storage elements, and dispersion occurs as modes of adjacent resonators are evanescently coupled. By contrast, in RGWNs, energy is not stored resonantly in discrete resonators, but rather in the network of waveguides that are designed to exhibit a collective resonant behaviour.

The operation of RGWNs was demonstrated in this chapter using plasmonics, which allowed for a simple layout and broadband range of operation; however, this implementation also brought about substantial attenuation due to the fundamental loss of plasmonic modes. As indicated above, the plasmonic MIM modes used here have typical propagation lengths of about 50 microns due to metal loss. Since the RGWN scope is broader than the field of plasmonics, it calls for an all-dielectric implementation to mitigate the losses brought on by plasmonics. Implementing RGWNs using photonic circuitry would also address the coupling loss associated with the difference in the modal overlaps between the plasmonic modes in the RGWN and the interfacing dielectric optics.

This new design paradigm is based on different underlying physics and thus opens up new directions for the design of artificial optical materials and devices. Since the RGWN design relies on the interference of local waves, we can use these accessible design parameters to program optical functions directly onto the network. Furthermore, the constraints on the propagation and coupling of the local waves in RGWNs allow for the device operation to reduce to a simple mathematical representation using S-matrix formalism. This allows for the network programming to take the form of an optimization procedure over a relatively small parameter space. The RGWN S-matrix representation was demonstrated here where the inputs were given and the S-matrix of device was designed to give a desired output (e.g., 
routing, mode converting). However, this formalism could be extended to different type of functions, such as sensing, in which the inputs are given and the output changes are monitored. In this chapter, dichroic and trichroic RGWN color routing was demonstrated as a proof of concept; however, incorporating more components into the RGWN and therefore increasing the possible degrees of freedom, could allow for more complex devices or alternatively for devices with enhanced performance. Furthermore, we exemplified the RGWN design paradigm using plasmonics, nesting a split element simply by intersecting waveguides, still the concept is broad and implementing the concept using photonic component could open new opportunities in the design of photonic circuitry devices.

\section{Acknowledgements}

This work was supported by the DOE 'Light-Material Interactions in Energy Conversion' Energy Frontier Research Center under grant DE-SC0001293 and by the National Science Foundation under the Graduate Research Fellowship Program.

\section{References}

Bozhevolnyi, S.I. (2006), Volkov, V.S., Devaux, E., Laluet J.Y., Ebbesen, T.W. Channel plasmon subwavelength waveguide components including interferometers and ring resonators. Vol.440, (2006), pp. 508-511

Fan, S. (1998), Villeneuve, P., Villeneuve, J., Haus, H.A. Channel drop filters in photonic crystals. Opt. Express, Vol.3, (1998), pp. 4-11

Feigenbaum, E (2007-1), Orenstein, M. Perfect 4-way splitting in nano plasmonic Xjunctions. Opt. Express, Vol.15, (2007), pp. 17948-17953

Feigenbaum, E (2007-2), Orenstein, M, Modeling of Complementary (Void) Plasmon Waveguiding, J. Lightwave Tech., Vol.25, (2007), pp. 2547- 2562

Feigenbaum, E. (2010). Atwater, H.A., Resonant Guided Wave Networks, Phys. Rev. Lett. Vol.104, (2010), 147402.

Feigenbaum, E. (2010-2), Burgos, S.p., Atwater, H.A. Programming of Inhomogeneous Resonant Guided Wave Networks. Opt. Express Vol.18, (2010), 25584-25595.

Little, B.E (1997), Chu, S.T., Haus, H.A., Foresi, J., Laine, J.P. Microring resonator channel dropping filters. J. Lightwave Tech., Vol.15, (1997), pp. 998-1005

Palik, E.D. (1998), Handbook of optical constants of solids, 2'nd Ed. San-Diego: Academic 1998.

Shalaev, V.M. (2007). Optical negative-index metamaterials. Nature Photon., Vol.1, (2007), pp. $41-48$

Smith, D.R. (2004), Pendry, J.B., Wiltshire, M.C.K. Metamaterials and Negative Refractive Index. Science, Vol.305, (2004), pp. 788-792

Yariv, A (1999), Yong L., R.K., Scherer, A. Coupled-resonator optical waveguide: a proposal and analysis. Opt. Lett., Vol.24, (1999), pp. 711-713 


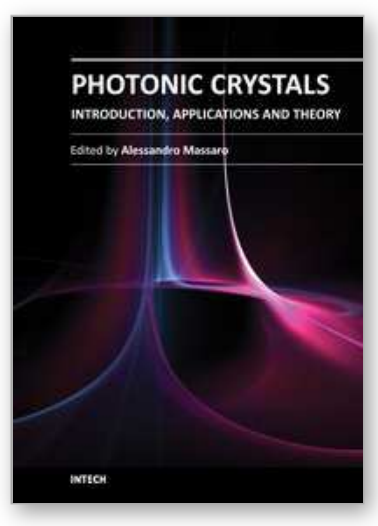

\author{
Photonic Crystals - Introduction, Applications and Theory \\ Edited by Dr. Alessandro Massaro
}

ISBN 978-953-51-0431-5

Hard cover, 344 pages

Publisher InTech

Published online 30, March, 2012

Published in print edition March, 2012

The first volume of the book concerns the introduction of photonic crystals and applications including design and modeling aspects. Photonic crystals are attractive optical materials for controlling and manipulating the flow of light. In particular, photonic crystals are of great interest for both fundamental and applied research, and the two dimensional ones are beginning to find commercial applications such as optical logic devices, micro electro-mechanical systems (MEMS), sensors. The first commercial products involving twodimensionally periodic photonic crystals are already available in the form of photonic-crystal fibers, which use a microscale structure to confine light with radically different characteristics compared to conventional optical fiber for applications in nonlinear devices and guiding wavelengths. The goal of the first volume is to provide an overview about the listed issues.

\title{
How to reference
}

In order to correctly reference this scholarly work, feel free to copy and paste the following:

Eyal Feigenbaum, Stanley P. Burgos and Harry A. Atwater (2012). Resonant Guided Wave Networks, Photonic Crystals - Introduction, Applications and Theory, Dr. Alessandro Massaro (Ed.), ISBN: 978-953-51-0431-5, InTech, Available from: http://www.intechopen.com/books/photonic-crystals-introduction-applications-andtheory/resonant-guided-wave-networks

\section{INTECH}

open science | open minds

\author{
InTech Europe \\ University Campus STeP Ri \\ Slavka Krautzeka 83/A \\ 51000 Rijeka, Croatia \\ Phone: +385 (51) 770447 \\ Fax: +385 (51) 686166 \\ www.intechopen.com
}

\author{
InTech China \\ Unit 405, Office Block, Hotel Equatorial Shanghai \\ No.65, Yan An Road (West), Shanghai, 200040, China \\ 中国上海市延安西路65号上海国际贵都大饭店办公楼 405 单元 \\ Phone: +86-21-62489820 \\ Fax: +86-21-62489821
}


(C) 2012 The Author(s). Licensee IntechOpen. This is an open access article distributed under the terms of the Creative Commons Attribution 3.0 License, which permits unrestricted use, distribution, and reproduction in any medium, provided the original work is properly cited. 